8th Alexander Friedmann International Seminar

on Gravitation and Cosmology

International Journal of Modern Physics: Conference Series

Vol. 3 (2011) 331-341

(C) World Scientific Publishing Company

DOI: $10.1142 /$ S2010194511001437

\title{
BLACK HOLE AS A SUPERCOLLIDER
}

\author{
O. B. ZASLAVSKII \\ Department of Physcis and technology, Kharkov V. N. Karazin National University, \\ 4 Svoboda Square, Kharkov, 61077, Ukraine \\ zaslav@ukr.net
}

Received 25 June 2011

Revised 7 July 2011

\begin{abstract}
Recently, it was found that in the vicinity of the black hole horizon of a rotating black hole two particles can collide in such a way that the energy in their centre of mass frame becomes infinite (so-called BSW effect). I give a brief review of basic features of this effect and show that this is a generic property of rotating black holes. In addition, there exists its counterpart for radial motion of charged particles in the charged black hole background. Simple kinematic explanation is suggested that is based on observation that all massive particles fall in two classes. In the first case (by definition, "usual particles"), the velocity approaches that of light on the horizon in the locally-nonrotating frame due to special relationship between the energy and the angular momentum. In the second case, it tends to some value less than speed of light. As a result, the relative velocity also tends to the speed of light with infinitely growing Lorentz factor.
\end{abstract}

Keywords: Black hole horizon; centre of mass; extremal horizons.

PACS numbers: 04.70.Bw, 97.60.Lf

\section{Introduction}

Quite recently, a new interesting effect was discovered in the Kerr background. ${ }^{1}$ It was obtained from basic equations of classical mechanics of particles moving in the given metric, so the nontrivial observation ${ }^{1}$ came as surprise. Namely, it was argued, that under certain conditions, the energy in the center-of-mass frame of two colliding particles can grow unbound, so a black hole acts as a supercollider This opens a window into a new physics including the possibility of unknown channels of reaction between elementary particles, with potential astrophysical applications such as elucidation of the nature of active nuclei in the Galaxy, ${ }^{2}$ etc. Now, this subject became a rather hot topic and provoked a series of subsequent papers. ${ }^{3-22}$

In the present contribution, I summarize the main features of the effect and give them a qualitative explanation. Apart from this, I show that, actually there are two areas of application of the effect under discussion - generic rotating black holes and nonrotating but charged black holes (both features can combine). In doing so, I make accent on the presence of the horizon that enables to give a unified explanation without relying on the concrete details of the metric. The fact that effect is inherent 
to a generic rotating black hole is especially important in the given context since physical significance of the effect under discussion implies the presence of matter (say, accretion disc) around the horizon, so the black hole, as usual in astrophysics, is "dirty".

The essence of the effect (called BSW effect according to the names of its authors $^{1}$ ) is intimately connected, as I will show, with the presence of the horizon. The first paper ${ }^{1}$ where the effect was discovered dealt with the extremal horizon. Meanwhile, it was observed ${ }^{13}$ that the acceleration to infinite energies can occur not only for extremal black holes (as was stated in Ref. 1) but also for nonextremal ones, and the distinction between two cases was traced in detail for the Kerr metric. This is important since there are astrophysical limitations on the proximity of the angular momentum of a black hole to the extremal value. ${ }^{23}$ In this sense, the aforementioned result ${ }^{13}$ enables, in principle, to evade this restriction and consider the effect not only for extremal black holes.

All this serves as motivation for the elaboration of general approach from which the key role of the horizon follows directly according to discussion below.

One reservation is in order. In the present contribution, the unbound energies in the center of mass frame are discussed. Therefore, one may ask what mechanism restricts this growth. One possible answer consists in that an infinite proper time is required to gain such energies (see details below). Another possible factor is the role of backreaction and radiation neglected here. Thus, at the present, there is no complete self-contained picture of the effect under discussion. Nonetheless, the essence of this effect is pure kinematic, so at least in the main approximation such an approach looks quite reasonable.

\section{Basic Formulas and Limiting Transitions}

Consider the generic axially symmetric metric. It can be written as

$$
d s^{2}=-N^{2} d t^{2}+g_{\phi \phi}(d \phi-\omega d t)^{2}+d l^{2}+g_{z z} d z^{2} .
$$

Here, the metric coefficients do not depend on $t$ and $\phi$. On the horizon $N=0$. Alternatively, one can use coordinates $\theta$ and $r$, similar to Boyer-Lindquist ones for the Kerr metric, instead of $l$ and $z$. In (1) we assume that the metric coefficients are even functions of $z$, so the equatorial plane $\theta=\frac{\pi}{2}(z=0)$ is a symmetry one.

In the space-time under discussion there are two conserved quantities $u_{0} \equiv-E$ and $u_{\phi} \equiv L$ where $u^{\mu}=\frac{d x^{\mu}}{d \tau}$ is the four-velocity of a test particle, $\tau$ is the proper time and $x^{\mu}=(t, \phi, l, z)$ are coordinates. The aforementioned conserved quantities have the physical meaning of the energy per unit mass (or frequency for a lightlike particle) and azimuthal component of the angular momentum, respectively. It follows from the symmetry reasonings that there exist geodesics in such a background which lie entirely in the plane $\theta=\frac{\pi}{2}$. Then, the first integrals for such geodesics read (dot denotes the derivative with respect to the proper time $\tau$ ):

$$
\dot{t}=u^{0}=\frac{E-\omega L}{N^{2}} .
$$


We assume that $\dot{t}>0$, so that $E-\omega L>0$ and

$$
\begin{gathered}
\dot{\phi}=\frac{L}{g_{\phi \phi}}+\frac{\left(-\omega^{2} L+E \omega\right)}{N^{2}}, \\
\dot{l}^{2}=\frac{(E-\omega L)^{2}}{N^{2}}-1-\frac{L^{2}}{g_{\phi \phi}} .
\end{gathered}
$$

Here, timelike geodesics are considered. For definiteness, we consider a pair of particles labeled by the subscript $i=1,2$ and having the equal rest masses $m_{1}=$ $m_{2}=m$. We also assume that both particle are approaching the horizon, so $i<0$ for each of them.

The quantity which is relevant for us is the energy in the centre of mass frame ${ }^{1-4}$ $E_{c . m .}=\sqrt{2} m \sqrt{1-u_{\mu(1)} u^{\mu(2)}}$. After simple manipulations, one obtains from (2)-(4) that

$$
\frac{E_{c . m .}^{2}}{2 m^{2}}=c+1-Y, c=\frac{X}{N^{2}},
$$

where

$$
X=X_{1} X_{2}-Z_{1} Z_{2}
$$

$X_{i} \equiv E_{i}-\omega L_{i}$

$$
\begin{gathered}
Z_{i}=\sqrt{\left(E_{i}-\omega L_{i}\right)^{2}-N^{2} b_{i}}, b_{i}=1+\frac{L_{i}^{2}}{g_{\phi \phi}}, \\
Y=\frac{L_{1} L_{2}}{g_{\phi \phi}} .
\end{gathered}
$$

Here, the crucial role is played by the quantity $c$ that determines whether the energy can grow unbound. Now, we will discuss different limiting transitions.

1) Let, for generic $L_{i}$, one approaches the horizon, so $N \rightarrow 0$. Expanding the radicals and retaining the first non-vanishing corrections in the numerator, one obtains (subscript " $H$ " refers to the horizon value):

$$
\begin{aligned}
& \left(\frac{E_{c . m .}^{2}}{2 m^{2}}\right)_{H}=1+\frac{b_{1(H)}\left(L_{2(H)}-L_{2}\right)}{2\left(L_{1 H}-L_{1}\right)}+\frac{b_{2(H)}\left(L_{(1) H}-L_{1}\right)}{2\left(L_{2(H)}-L_{2}\right)}-\frac{L_{1} L_{2}}{\left(g_{\phi \phi}\right)_{H}}, \\
& L_{i(H)} \equiv \frac{E_{i}}{\omega_{H}} .
\end{aligned}
$$

By a very meaning of derivation, it is supposed in (9) that $L_{1} \neq L_{2(H)}, L_{2} \neq L_{2(H)}$.

Let us now specify the range of angular momenta in such a way that one of them is close to the critical value: $L_{1}=L_{1(H)}(1-\varepsilon), \varepsilon \ll 1, L_{2} \neq L_{2(H)}$. Then we have that

$$
\left(\frac{E_{c . m .}^{2}}{2 m^{2}}\right)_{H} \approx \frac{b_{1(H)}\left(L_{2(H)}-L_{2}\right)}{2 L_{1(H)} \varepsilon}
$$


This quantity can be made as large as one likes due to $\varepsilon \rightarrow 0$. It follows from (5) and (9) that

$$
\lim _{L_{1} \rightarrow L_{1(H)}} \lim _{N \rightarrow 0} E_{c . m .}=\infty
$$

2) Let us take $L_{1} \rightarrow L_{1(H)}$ first and, then, consider the limit $N \rightarrow 0$. The previous formula (9) is valid both for nonextremal and extremal horizons. In contrast to it, now the distinction between two types of the horizon comes into play.

2a) First, consider the nonextremal case. We are interested in the immediate vicinity of the horizon where the effect under discussion is expected to show up. Near the horizon, we can infer the restriction that follows from the condition of positivity of the expression inside the square root in (7). To this end, let us use the general form of the asymptotic expansion for the metric coefficient $\omega$ that follows from the general requirement of regularity of the geometry near the nonextremal horizon: ${ }^{24}$

$$
\omega=\omega_{H}+B N^{2}+\ldots
$$

Here, $\omega_{H}$ is constant and has the physical meaning of the angular velocity of the horizon itself, the coefficient $B=B(\theta)$. For the case $\theta=\frac{\pi}{2}$ under consideration, $B$ is simply constant. Its exact value is model-dependent.

Then, the condition of the positivity of (4) cannot be satisfied since the first term has the order $N^{2}$ whereas the others have the order $N^{0}$ and are negative. It means that the horizon is unreachable (admissible region adjacent to the horizon shrinks to the point and there is some turning point situated on a finite distance from the horizon). Therefore, the present case should be rejected.

2b) Now, consider the extremal horizon. Then, instead of (12), one has more general expansion

$$
\omega=\omega_{H}-B_{1} N+B_{2} N^{2}+\ldots
$$

The distinction between expansions for both horizons can be understood using the Kerr metric as an example. The first corrections have the order $r-r_{H}$ where $r$ is the Boyer-Lindquist coordinate. However, for the nonextremal case $N^{2} \sim r-r_{H}$ whereas for the extremal Kerr metric $N^{2} \sim\left(r-r_{H}\right)^{2}, B_{1}=M^{-1}$ where $M$ is the mass.

In a more general case, one can just appeal to the definition of the nonextremal and extremal black holes using the proper length. Namely, in the nonextremal case $N \approx \kappa l$ near the horizon where $\kappa$ is the surface gravity and in the extremal one $N \approx N_{0} \exp (-A l)$, with $N_{0}, A=$ const $>0$ and $l \rightarrow \infty$. In principle, so-called ultraextremal horizons with $N \sim l^{-s}, s>0$ are also possible which can contain fractional powers of $r-r_{H}$ (where $r$ is the analogue of the Boyer-Lindquist coordinate) but we do not discuss them here. (For the spherically-symmetric configurations such horizons are classified in Ref. 25.) 
After the substitution of (32) to (6), (7), we obtain after simple manipulations that

$$
\frac{E_{c . m .}^{2}}{2 m^{2}} \approx \frac{\left(E_{2}-\omega_{H} L_{2}\right)}{N}\left[B_{1} \frac{E_{1}}{\omega_{H}}-\sqrt{\left(\frac{E_{1}^{2}}{\omega_{H}^{2}} B_{1}^{2}-b_{1}\right)}\right] .
$$

Here, it is implied that the condition of the positivity is fulfilled for the expression inside the radical (this cannot be worked out in more detail in a model-independent way). Thus,

$$
\lim _{N \rightarrow 0} \lim _{L_{1} \rightarrow L_{1(H)}} E_{c . m .}=\infty .
$$

The extremal case has one more interesting feature. Namely, the proper time needed to reach the horizon, tends to infinity. Indeed, it follows from (4) and (32), that for the particle having $L=L_{(H)}$ and approaching the horizon,

$$
\tau \sim \int \frac{d l N}{Z} \sim l \rightarrow \infty
$$

since the proper distance from any point to the extremal horizon is infinite. For the nonextremal horizon the proper distance is finite as well as the proper time. Also, one can easily find from (3), (4) that the number of revolutions

$$
\Delta \phi \approx \frac{E B_{1}}{\sqrt{\left(\frac{E^{2}}{\omega_{H}^{2}} B_{1}^{2}-b_{1}\right)}} \int \frac{d l}{N} .
$$

Using again the asymptotic form $N \approx N_{0} \exp (-A l)$ in the extremal case, we see that $\Delta \phi \rightarrow \infty$.

3) In two previous situations, the result was formally determined by a play between two small quantities $\varepsilon$ and $N$ and the order of taking the limits $\varepsilon \rightarrow$ 0 and $N \rightarrow 0$. Meanwhile, it is of interest to trace, what happens when both quantities are small but nonzero, and what are limitations on the possibility of collision with infinitely growing energies. For the Kerr metric, a particle with the critical value of the angular momentum $L_{(H)}$ cannot come from infinity since a potential barrier prevents this, so that the energy cannot grow unbound as a result of single scattering. ${ }^{1-4}$ Meanwhile, as was demonstrated for the Kerr metric, ${ }^{13}$ this becomes possible if multiple scattering occurs, so one of colliding particles does not come from infinity but receives the near-critical angular momentum as a result of collision near the horizon. As far as the generic spacetime is concerned, in principle it can happen that a particle with the critical angular momentum coming from infinity is able to reach the horizon. However, such a possibility is model-dependent and requires special conditions on the behavior of the metric. Meanwhile, we are interested in features that have general model-independent character. Therefore, we will not discuss such particular cases and assume that a particle has a near-critical angular momentum in the near-horizon region just due to multiple collisions. Let us see the necessary condition for this. 
From the condition $Z^{2} \geq 0$ where $Z$ is defined in (7) we obtain for the nonextremal horizon using (12) that the process under discussion can indeed occur but only in the narrow strip near the horizon where

$$
0 \leq N \leq \frac{E \varepsilon}{\sqrt{b_{H}}}
$$

Thus, the energy (10) can be indeed as large as one likes but this happens provided a particle acquires the near-critical angular momentum in the region bounded by Eq. (18). If $\varepsilon=0$ exactly, the permitted strip shrinks to the point and we returned to case $2 \mathrm{a}$ when the effect is impossible. For the extremal horizon, there is no limitation similar to (18) since one can put $\varepsilon=0$ in accordance with case $2 \mathrm{~b}$ ), see also Eq. (32).

4) For completeness, let us consider the case when simultaneously $L_{1}=$ $L_{1(H)}, L_{2}=L_{2(H)}$. In the nonextremal case it follows from (12) that the radical cannot remain positive near the horizon and the horizon is unreachable, so this case is irrelevant for our analysis. For the extremal one, in the horizon limit we obtain from (32) that the terms of the order $N$ in the numerator cancel, so that the first non-vanishing term has the same order $N^{2}$ as the denominator. As a result, the quantity $E_{c . m}$. is finite. However, the proper time needed to reach the horizon is still infinite.

If we compare the meaning of limits 1 and 2 , we see that in the nonextremal case the energy in the centre of mass frame is finite but can be made as large as one likes if the angular momentum of one of two colliding particles is chosen arbitrarily close to the critical value. If this value is chosen exactly equal to the critical value from the very beginning, the energy can be made as large as one wishes when one approaches the horizon (it becomes possible in the extremal case only). This is direct generalization of observations made in Refs. 1 and 13 for the Kerr metric.

\section{Charged Black Holes}

It turns out that the BSW effect exists even for pure radial motion in the metric of spherically-symmetric black hole. Consider the Reissner-Nordström black hole $d s^{2}=-d t^{2} f+\frac{d r^{2}}{f}+r^{2} d \omega^{2}$. Here $d \omega^{2}=\sin ^{2} \theta d \phi^{2}+d \theta^{2}, f=1-\frac{2 M}{r}+\frac{Q^{2}}{r^{2}}$ where $M$ is the black hole mass, $Q$ is its charge. For radial motion, repeating derivation step by step, one obtains that

$$
\frac{E_{c m}^{2}}{2 m^{2}}=1+\frac{X_{1} X_{2}-Z_{1} Z_{2}}{f m^{2}},
$$

where

$$
X_{i}=E_{i}-\frac{q_{i} Q}{r}, Z_{i}=\sqrt{X_{i}^{2}-m^{2} f}
$$

The analogy with the rotaitonal case (5) - (7) becomes obvious, the role of the anhular momentum is played by charge. Thus, the analogue of the BSW is indeed present here. 


\section{Kinematic Explanation}

Previous consideration was based on straightforward calculations and careful evaluation of the asymptotic form of the scalar product of two fur-velocites near the horizon. In the present section, I give general qualitative kinematic explanation in the terms of particles' three-velocities. In Ref. 1 the remark has been made by passing that the effect is connected with a crucial difference between kinematics of "usual" and "critical" particles. In the first case, a particle hits the horizon of a rotating black hole perpendicularly, in the second one it does it at some incident angle. This important observation does not give, however, the full explanation of the phenomenon. For example, the effect exists even for pure radial motion of charged particles in the Reissner-Nordström space-time ${ }^{13}$ when, obviously, all particle approach horizon perpendicularly. From another hand, two different critical particles can collide at some nonzero angle if they have different momenta. However, this does not produce the infinite energy in the centre of mass. Thus, an interesting property mentioned in Ref. 1 is neither necessary nor sufficient for the explanation of the effect under discussion.

In what follows we will use the tetrad basis. Denoting coordinates $x^{\mu}$ as $x^{0}=$ $t, x^{1}=l, x^{2}=z, x^{3}=\phi$, we choose the tetrad vectors $h_{(a) \mu}$ in the following way: $h_{(0) \mu}=-N(1,0,0,0), \quad h_{(1) \mu}=(0,1,0,0), h_{(2) \mu}=\sqrt{g_{z z}}(0,0,0,1), h_{(3) \mu}=$ $\sqrt{g_{\phi \phi}}(-\omega, 0,0,1)$. If such a tetrad is attached to an observer moving in the metric (1), it has meaning of zero angular momentum observer (ZAMO). ${ }^{26}$ They are "rotate with the geometry" in the sense that $\frac{d \phi}{d t} \equiv \omega$ for them. The advantage of using the tetrad components consists in that one can use the formulas of special relativity in the flat space-time tangent to any given point.

Then, we can introduce the three-velocity according to

$$
v^{(i)}=v_{(i)}=\frac{u^{\mu} h_{\mu(i)}}{-u^{\mu} h_{\mu(0)}} .
$$

From equations of motion (2) - (4) and formulas for tetrad components, we obtain

$$
\begin{gathered}
v^{(3)}=\frac{L N}{\sqrt{g_{\phi \phi}}(E-\omega L)}, \\
v^{(1)}=\sqrt{1-\frac{N^{2}}{(E-\omega L)^{2}}\left(1+\frac{L^{2}}{g_{\phi \phi}}\right) .}
\end{gathered}
$$

Then, introducing also the absolute value of the velocity $v$ according to

$$
v^{2}=\left[v^{(1)}\right]^{2}+\left[v^{(2)}\right]^{2}
$$

one can find that

$$
E-\omega L=\frac{N}{\sqrt{1-v^{2}}},
$$




$$
v^{2}=1-\left(\frac{N}{E-\omega L}\right)^{2} .
$$

\section{Limiting Transitions for Relative Velocity}

The energy $E_{c . m}$. in the centre of mass frame of two colliding particles

$$
E_{c . m .}^{2}=-\left(p_{1}^{\mu}+p_{2}^{\mu}\right)\left(p_{1 \mu}+p_{2 \mu}\right)=m_{1}^{2}+m_{2}^{2}-2 m_{1} m_{2} u_{1}^{\mu} u_{2 \mu} .
$$

Here, $p_{i}^{\mu}=m_{i} u_{i}^{\mu}(i=1,2)$ is the four-momentum of each particle, $m_{i}$ are their rest masses. By definition, this is a scalar which can be calculated in any frame. It is convenient to use a frame comoving with respect to one of colliding particles (say, particle 2). If one uses tetrad representation, one can exploit formulas known in a flat space-time. Then, the quantity of interest is

$$
\gamma=-u_{1}^{\mu} u_{2 \mu}=\frac{1}{\sqrt{1-w^{2}}}
$$

where $w$ is, by definition, their relative velocity (which in this frame coincides with the velocity of particle 1), $\gamma$ has the meaning of the Lorentz factor.

The effect of unbound energies occurs if $w \rightarrow 1$, so $\gamma \rightarrow \infty$.

Now, I remind some simple formulas from special relativity. If in the laboratory frame particle 1 has the velocity $\vec{v}_{1}=v_{1} \vec{n}_{1}$ and particle 2 has the velocity $\vec{v}_{2}=v_{2} \vec{n}_{2}$, the value of the relative velocity is equal to

$$
w^{2}=1-\frac{\left(1-v_{1}^{2}\right)\left(1-v_{2}^{2}\right)}{\left[1-v_{1} v_{2}\left(\vec{n}_{1} \vec{n}_{2}\right)\right]^{2}} .
$$

This formula can be found in textbooks (see. e.g., problem 1.3. in Ref. 27). Now, we enumerate different limiting transitions for this quantity relevant in our context.

a) $v_{1} \rightarrow 1, v_{2}<1,\left(\vec{n}_{1} \vec{n}_{2}\right)$ is arbitrary.

It is obvious from (29) that in this case $w \rightarrow 1$ independent of the quantity $\left(\vec{n}_{1} \vec{n}_{2}\right)$. This corresponds to the well known fact that the velocity of light $c$ is always equal to 1 (in geometrical units) in any frame.

b) $v_{1} \rightarrow 1, v_{2} \rightarrow 1$ in such a way that $v_{i}=1-A_{i} \delta$ where $A_{i}(i=1,2)$ are constants, $\delta \ll 1$.

b1) If $\left(\vec{n}_{1} \vec{n}_{2}\right) \neq 1$, it is seen from (29) that

$$
w^{2} \approx 1-\frac{4 A_{1} A_{2} \delta^{2}}{\left[1-\left(\vec{n}_{1} \vec{n}_{2}\right)\right]^{2}},
$$

so we have $v \rightarrow 1$ again.

b2) If $\left(\vec{n}_{1} \vec{n}_{2}\right)=1$, the situation changes radically. Then,

$$
w \approx \frac{\left|A_{1}-A_{2}\right|}{A_{1}+A_{2}}<1 .
$$

c) $v_{1}<1, v_{2}<1,\left(\vec{n}_{1} \vec{n}_{2}\right)$ is arbitrary. Then, it is obvious that $w<1$. By itself, this case is trivial. However, it plays nontrivial role in the context under consideration (see below). 


\section{Asymptotics Near Horizon}

Let us now look what happens to particles' velocities near the horizon. For an usual particle, $E-\omega_{H} L \neq 0$ and it follows from (25) that in the horizon limit $N \rightarrow 0$, $v \rightarrow 1$. Apart from this, it follows from (22), (23) that in this limit $v^{(3)} \rightarrow 0$, $v^{(1)} \rightarrow 1$. Therefore, the unit vector $\vec{n}$ is pointed along the $l$ direction, so for any two such particles $\left(\vec{n}_{1} \vec{n}_{2}\right)=1$. However, for a critical particle, the situation is different.

At first, consider the extremal horizon. Then, near it, we have an expansion

$$
\omega=\omega_{H}-B_{1} N+B_{2} N^{2}+\ldots
$$

For example, for the Kerr metric $B_{1}=M^{-1}$ where $B$ is the black hole mass. ${ }^{12}$ We obtain from (25) that

$$
v^{2}=1-\frac{1}{L^{2} B_{1}^{2}}<1
$$

Apart from this, in the critical case the quantities $v^{(1)}$ and $v^{(3)}$ have the same order, so a particle hits the horizon at some nonzero angle with respect to the normal direction in accordance with the remark made in 1 . Correspondingly, $\left(\vec{n}_{1} \vec{n}_{2}\right) \neq 1$. Now, using the above properties, we can enumerate different types of collisions near the horizon.

\subsection{Collision between two usual particles}

This situation corresponds to case b2). Then, it follows form (31) that $w<1$, the Lorentz factor $\gamma$ is finite, so the effect of infinite energies is absent.

\subsection{Collision between two critical particles}

This situation corresponds to case $\mathrm{c}$ ). Then, we have that $w<1$, so the effect under discussion is also absent.

\subsection{Collision between an usual (1) and critical (2) particles}

This type of collision falls into the class a) described above. As a result, we have $w \rightarrow 1, \gamma \rightarrow \infty$ and the effect of infinite acceleration is present. The fact that $v_{2}<1$ explains why critical particle cannot reach the extremal horizon for finite proper time, in accordance with Refs. 7 and 12. Indeed, the proper distance is infinite, so the proper time for a particle 2 having $v_{2}<1$ everywhere on its trajectory is certainly infinite.

In the nonextremal case a near-critical particle cannot reach the horizon ${ }^{12,24}$ since $\omega-\omega_{H} \sim N^{2}$ when $N \rightarrow 0$, so the right hand side of (4) cannot be positive. However, it can approach the horizon as nearly as one likes. Let $E=\omega_{H} L(1+\delta)$, $\delta \ll 1$. Then, we must keep $\delta$ such have that $\delta \gtrsim N$ to ensure the positivity of $i^{2}$ in (4). Let $\delta=A N(P)$ where $A$ is some finite coefficient, $P$ is the point of collision. 
Then, $1-v^{2}=\left(\frac{N}{E-\omega_{H} L}\right)^{2} \approx \frac{1}{\left(\omega_{H} L A\right)^{2}} \neq 0$. Thus, taking the point of collision closer and closer to the horizon and simultaneously taking the energy closer and closer to the critical value, we can gain $v<1$ and, thus, the effect of infinite acceleration for the energy in the centre of mass for collision between an usual and critical particles. However, this requires multiple scattering since, say, for the Kerr metric, such a particle cannot come from infinity. Apart from this, the collision should occur in a narrow strip near the horizon (see Refs. 7 and 12 for details).

\section{Discussion and Conclusions}

Thus, we suggested very simple and direct derivation of the effect of growing energy from first principles and even without using the explicit form of the black hole metric, valid for a generic rotating black hole. For massless particles explanation somewhat changes ${ }^{20}$ but it is also based on division to two classes of particles (with finite or infinite energy near the horizon).

There are important issues left beyond the scope of the present work. The BSW effect is obtained in the framework of the test particle approximation. Whether and how backreaction and gravitational radiation can affect the results deserves further treatment. Also, our "supercollider" acts in the frame of the centre of mass of colliding particles, an observer at infinity would see the outcome from the reaction of collision red-shifted, with finite energy. Nonetheless, collisions with the energy infinitely growing in the centre of mass frame lead to the appearance of qualitative new reactions that can, in principle, probe the Planck scale and have unexpected astrophysical consequences.

\section{Acknowledgment}

I thank the Organizing Committee of the Eighth Friedmann Seminar for support that made my participation possible and stimulating working atmosphere during the time of the conference.

\section{References}

1. M. Banados, J. Silk and S. M. West, Phys. Rev. Lett. 103, 111102 (2009).

2. A. A. Grib and Yu. V. Pavlov, Mod. Phys. Lett. A 23, 1151 (2008).

3. E. Berti, V. Cardoso, L. Gualtieri, F. Pretorius and U. Sperhake, Phys. Rev. Lett. 103, 239001 (2009).

4. T. Jacobson and T. P. Sotiriou, Phys. Rev. Lett. 104, 021101 (2010).

5. K. Lake, Phys. Rev. Lett. 104, 211102 (2010).

6. K. Lake, Phys. Rev. Lett. 104, 259903(E) (2010).

7. A. A. Grib and Yu.V. Pavlov, Pis'ma v ZhETF 92, 147 (2010) [JETP Letters 92, 125 (2010)].

8. S.-W. Wei, Y.-X. Liu, H. Guo and C.-E. Fu, Phys. Rev. D 82, 103005 (2010).

9. S.-W. Wei, Y.-X. Liu, H.-T. Li and F.-W. Chen, JHEP 12, 066 (2010).

10. P.-J. Mao, R. Li, L.-Y. Jia and J.-R. Ren, Acceleration of particles in EinsteinMaxwell-Dilaton black hole, arXiv:1008.2660v1. 
11. A. A. Grib and Yu. V. Pavlov, Grav. Cosmol. 17, 42 (2011).

12. O. B. Zaslavskii, Phys. Rev. D 82, 083004 (2010).

13. O. B. Zaslavskii, Pis'ma ZhETF 92, 635 (2010) [JETP Lett. 92, 571 (2010)].

14. M. Bañados, B. Hassanain, J. Silk and S. M. West Phys. Rev. D 83, 023004 (2011).

15. M. Kimura, K.-I. Nakao and H. Tagoshi, Phys. Rev. D 83, 044013 (2011).

16. T. Harada and M. Kimura, Phys. Rev. D 83, 024002 (2011).

17. T. Harada and M. Kimura, Phys. Rev. D 83, 084041 (2011).

18. Y. Zhu, S.-F. Wu, Y.-X. Liu and Y. Jiang, General stationary charged black holes as charged particle accelerators, arXiv:1103.3848v1.

19. O. B. Zaslavskii, Class. Quant. Grav. 28, 105010 (2011).

20. O. B. Zaslavskii, Phys. Rev. D 84, 024007 (2011).

21. A. A. Grib and Yu.V. Pavlov, Astropart. Phys. 34, 581 (2011).

22. A. A. Grib, Yu. V. Pavlov and O. F. Piattella, High energy processes in the vicinity of the Kerr's black hole horizon, arXiv:1105.1540v1.

23. K. S. Thorne, Astrophys. J. 191, 507 (1974).

24. A. J. M. Medved, D. Martin and M. Visser. Phys. Rev. D 70, 024009 (2004).

25. K. A. Bronnikov, E. Elizalde, S. D. Odintsov and O. B. Zaslavskii, Phys. Rev. D 78, 064049 (2008).

26. J. M. Bardeen, W. H. Press and S. A. Teukolsky, Astrophys. J. 178, 347 (1972).

27. A. P. Lightman, W. H. Press, R. H. Price and S. A. Teukolsky. Problem Book in Relativity and Gravitation (Princeton University Press, Princeton, 1975). 